459 EVALUATION OF THE EFFECT OF INTRAUTERINE INJECTION OF PLATELET-RICH PLASMA ON THE PREGNANCY RATE OF PATIENTS WITH A HISTORY OF IMPLANTATION FAILURE IN THE IN VITRO FERTILIZATION CYCLE

Marzieh Ghasemi. Zahedan University of Medical Sciences

\subsection{6/ijgc-2020-ESG0.86}

Introduction/Background Implantation failure is a major problem in reproductive medicine, and despite the various methods described for treatment, there is little consensus on the most effective method. Therefore, this study was conducted to investigate the effect of intrauterine injection of platelet-rich plasma (PRP) on the pregnancy rate of patients with a history of implantation failure in the in vitro fertilization (IVF) cycle. Methodology In this clinical trial study, women attending the infertility clinic of Ali ibn Abitaleb Hospital in Zahedan (Iran) in 2019, who had a history of implantation failure and were candidates for frozen embryo transfer (FET), were examined. After receiving informed consent, the patients were divided into two groups of PRP recipients and the control group. IVF was performed routinely, and in the PRP receiving group, intrauterine injection was performed 48 hours before embryo transfer (ET). Then, demographic factors such as age, body mass index (BMI) and endometrial thickness were investigated in the two groups. The number of gestational sacs, the rate of implantation, the frequency of chemical and clinical pregnancies, as well as the frequency of abortion were compared in two groups.

Results In this study, 90 patients with a history of implantation failure participated the study and finally the information of 85 patients was studied. The mean age of the patients as well as the BMI did not differ between the two groups. The frequency of chemical pregnancy was $40 \%$ in the experimental group, $27 \%$ in the control group, and regarding clinical pregnancy $33 \%$ in the experimental group, and $24 \%$ in the control group, but there was no significant difference between the two groups. The rate of implantation, the mean thickness of the endometrium and the frequency of abortion did not differ significantly between the two groups.

Conclusion In general, the results of this study showed that in patients with endometrial thickness greater than $8 \mathrm{~mm}$ with a history of recurrent implantation failure, intrauterine injection of PRP had no effect on fertility outcome.

Disclosures None.

\section{ONCOLOGICAL AND FERTILITY OUTCOMES AFTER VAGINAL RADICAL TRACHELECTOMY: SINGLE CENTRE RETROSPECTIVE ANALYSIS}

Iza Drobne Predanič, Luka Kovač, Borut Kobal, Leon Meglič. University Medical Centre Ljubljana; Division of Gynaecology; Department of Gynaecology

\subsection{6/ijgc-2020-ESG0.87}

Introduction/Background Trachelectomy with pelvic lymphadenectomy is considered a viable surgical procedure for fertility preservation in patients with early stage cervical carcinoma. The purpose of this study was to analysis postprocedural follow-up of oncological and fertility outcomes at a single centre.

Methodology We conducted a retrospective analysis of women with early stage cervical carcinoma, treated at the University Medical Centre Ljubljana, between 2007 and 2017. The study group was compiled of 26 women, age 22 to 40 years old (mean 32,8 years old).

Results Out of 26 women with early stage cervical cancer, 25 women were treated with vaginal radical trachelectomy (VRT) and laparoscopic pelvic lymphadenectomy (LPL) and one with only VRT. Mean age and BMI were 32,8 years and $21,54 \mathrm{~kg} /$ $\mathrm{m} 2$; respectively. One patient was IA1, two IA2, twenty-two IB1 and one IB2. Histology subtypes included squamous cell carcinoma $(n=16)$, adenocarcinoma $(n=8)$, endometrioid adenocarcinoma $(n=1)$ and clear cell carcinoma $(n=1)$. No perioperative complications were documented, postoperative complications included urinary retention and anaemia. All were treated with conservative measures. Within the follow-up period (12 - 60 months) 3 women (12\%) developed disease recurrence, all were treated with local excision and adjuvant chemoradiotherapy. One patient died 15 months after primary treatment, following progression of the disease. Two remaining patients are in remission. Of the 24 women who had neither additional surgical procedures, nor adjuvant therapy, we documented 14 attempted pregnancies. All women with successful pregnancies had a separate procedure; laparoscopic cerclage. Out of 14 pregnancies five ended in miscarriage, one pregnancy resulted in second trimester delivery (24/25. week) and eight resulted in third trimester delivery (34.-38. week). All births were performed with a Caesarean section.

Conclusion Cervical carcinoma effects women in reproductive age, limiting their chance for a successful pregnancy. Radical trachelectomy with SNL or pelvic lymphadenectomy should be an option for women that wish to preserve fertility. Good preoperative staging is essential in the process. Our data suggest that surgical approach is associated with low perioperative morbidity. Our recorded pregnancy rate is similar to that described in the literature.

Disclosures Authors have nothing to disclose.

\section{FERTILITY-SPARING TREATMENT FOR EARLY STAGE CERVICAL CANCER: A SINGLE CENTER EXPERIENCE WITH CONIZATION AND NODAL EVALUATION}

${ }^{1}$ Fabio Martinelli, ${ }^{2}$ Antonino Ditto, ${ }^{3}$ Francesca Filippi, ${ }^{2}$ Giorgio Bogani, ${ }^{2}$ Umberto Leone Roberti Maggiore, ${ }^{2}$ Mariateresa Evangelista, ${ }^{2}$ Mauro Signorelli, Valentina Chiappa, ${ }^{2}$ Salvatore Lopez, ${ }^{3}$ Edgardo Somigliana, ${ }^{2}$ Francesco Raspagliesi. ${ }^{1}$ Fondazione Irccs Istituto Nazionale Tumori, Milan, Italy; Gynecologic Oncology; ${ }^{2}$ Fondazione Irccs Istituto Nazionale Tumori, Milan, Italy; ${ }^{3}$ Fondazione Irccs Ca' Granda Ospedale Maggiore Policlinico, Milan, Italy

\subsection{6/ijgc-2020-ESGO.88}

Introduction/Background To evaluate oncological and obstetrical outcomes of early stage cervical cancer patients who attempted a conservative management to retain their childbearing potential.

Methodology Data of women (aged $<40$ years) who attempted a fertility sparing treatment for FIGO stage IA1-LVSI+, IB1 and IB2 cervical cancer were prospectively collected. All patients underwent cervical conization/s and laparoscopic nodal evaluation (pelvic lymphadenectomy/sentinel node mapping). Oncological and obstetrical outcomes were assessed.

Results Overall, 44 patients met the inclusion criteria. Fortyone (93.2\%) women were nulliparous. There were: $3(6.8 \%)$ IA1-LVSI+; 11 (25\%) IA2; 25 (56.8\%) IB1; 5 (11.4\%) IB2 cervical cancers, according to 2018 FIGO stage. Hystological type were: $25(56.8 \%)$ squamous carcinoma; 18 (40.9\%) adenocarcinoma and $1(2.3 \%)$ adenosquamous carcinoma. 